\title{
Production Monitoring and Process Improvement for Floor Panel Manufacturing
}

\author{
Chelsea RITTER ${ }^{1 *}$, Xinming LI $^{2}$ and Mohamed AL-HUSSEIN ${ }^{3}$ \\ ${ }^{1}$ M.Sc. Student, Department of Civil and Environmental Engineering, University of Alberta \\ ${ }^{2}$ Ph.D. Candidate, Department of Civil and Environmental Engineering, University of Alberta \\ ${ }^{3}$ Professor, Department of Civil and Environmental Engineering, University of Alberta \\ "Corresponding author's e-mail: critter1@ualberta.ca
}

\begin{abstract}
Panelized home construction allows for the construction of homes to be completed in a factory, but in only two dimensions, compared to the three dimensional module that is produced in modular construction facilities. Keeping the panels detached until they reach the final destination permits for more efficient transport of panels and allows the factory to be divided into more specialized areas. This paper presents a case study of an established panelized home manufacturer, where the floor production area is identified as an area for potential process improvement. Possible areas for process improvement are identified by conducting a time study, carrying out observation, and constructing a simulation model in which potential process improvements can be tested. Opportunities are identified for process improvement, and the anticipated results of implementing certain changes are quantified through the use of simulation in order to aid management in making decisions regarding which changes are to be implemented and in what order. Some possible areas for improvement of the floor production area, including reducing the waiting time for the multi-function bridge by manually applying glue, aligning the joists in the correct orientation prior to their reaching the floor jig to eliminate the need to rotate the joists, and installing a bridge for sheathing board delivery that eliminates the time spent walking to retrieve the sheathing boards.
\end{abstract}

\section{KEYWORDS}

Panelized Construction; Time Study; Numerical Simulation; Process Improvement

\section{INTRODUCTION}

\section{Literature Review}

Many of the time studies and process improvement studies that are done in modular construction manufacturing focus on total task times or station times. One such study was carried out by Moghadam (2014) at a different modular home manufacturing facility, also located in Edmonton, Alberta. This study focused on the application of lean tools to the manufacturing process, and included time studies of station times to assist in production levelling. Some of these station categories included the floor construction time, wall framing time, and roof framing time. Moghadam recommends the use of multi-skilled labour to level the production time between 
stations. This labour is able to work at any station and can move to where they are needed in order to keep the station times as equal as possible. While this is a good solution, there is also the opportunity to improve the work processes and setup at each station in order to reduce the times, where required, without the necessity for increased labour at a station by further investigating the individual tasks required to complete the work at each station.

An earlier study of the floor construction area in the same factory was carried out by Inyang et al. (2012). The setup of the area was substantially different at that time. The paper describes only one table, from which four floor panels are constructed each day by two carpenters. There was no automation at that time (as opposed to the current state today that utilizes an MFB). While the study did touch on the productivity of the process, the main focus was on improving the ergonomics involved with the process. This is important, as often the fastest or most effective way to do something is also the safest. Since the time of this study the process and layout used in the area have changed significantly. This has created the necessity for another study with an aim to approve the productivity and analyse the current work process. The earlier study also recognizes the necessity to account for work interruptions and other non-value added activities, and the stochastic nature of the activity durations; however, this was not included in the study.

Ikuma et al. (2011) studied a modular home factory in the United States with a similar approach, but with a very large focus on safety as well. They recognize that safety and productivity are linked and that improvements in one will bring about improvements in the other. They also looked at the specific tasks that the workers were doing in order to determine the percentage of time spent on value added and non value added activities. The plant that was investigated in their study had a lower level of automation than the plant to be studied in this paper, so this dynamic will change the results of the study as automation creates time where workers will either wait or carry out other tasks.

\section{Floor Production Line Setup}

In this panelized home construction facility in Edmonton, Alberta, Canada, the floor production line was identified as having a lower productivity than the rest of the plant. In the current state, a night shift is required in the floor production area if the rest of the plant is at full production. The floor production area consists of two tables with moving pins that indicate to the carpenter where the joists are to be placed. There is one multi-function bridge (MFB) that is able to work on either of the tables. One of the tables and the MFB are shown in Figure 1. The MFB can apply glue to the top of the joists, nail in the sheathing boards, route holes for the straps for the crane to be hooked through, use a saw to cut off excess sheathing, and mark where the walls will be erected once the panels are on site. Either one or two carpenters will lay out the joists and nail them together, and place the sheathing boards on top of the joists once the glue is applied. Once the panel is complete, the overhead crane is used to transfer the panels from the table to the trailers, which will be used to deliver the panels to the site. 


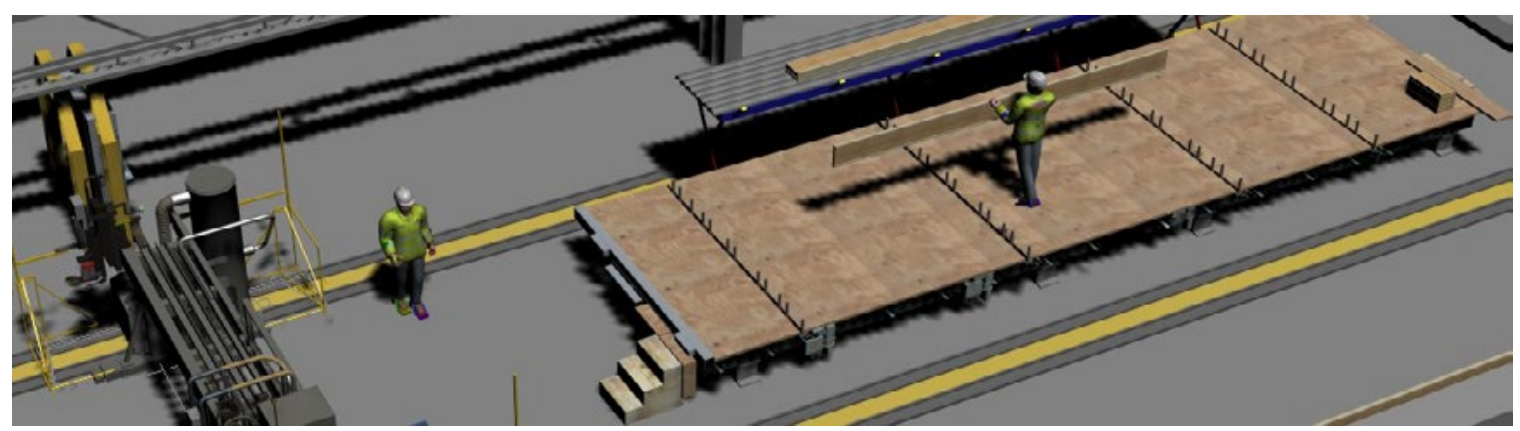

Figure 1. One of the two tables on which floor panels are constructed.

\section{Goals}

By completing this study, the management at the factory hope to be able to increase their production to a level equal to that achieved by the rest of the factory, which would allow them to eliminate the night shift that is required when the factory is producing at its maximum rate; this is equivalent to about 24 panels per day, compared to the 12 to 14 panels that are currently produced per day. The productivity improvements will be achieved both by implementing more efficient work practices for the construction of the floor panels, as well as by reducing the time spent on indirectly productive activities.

\section{METHODS}

\section{Study of Directly Productive Tasks}

In order to propose improvements to the floor construction area of the plant, a study of the current state is necessary. There are two ways that this is approached: a study of the time it takes to complete individual tasks, both in general and with a detailed breakdown, (the directly productive tasks) and a study of the time that is spent doing things that do not directly contribute to the construction of the floor panels (the indirectly productive tasks). The study of the directly productive tasks was carried out first, as a study of the time each task that contributes to the production of the floor panels takes is useful both for identifying the tasks that take longer than expected, and for use by the planning staff at the plant to verify the durations used for setting schedules. It is also useful to gain an understanding of the process and the sequence in which tasks are carried out.

Current State Simulation Model. The simulation model was constructed using the discrete event simulation tools in Simphony.NET, a program developed by AbouRizk and Mohamed (2000). For the current state simulation, the collected data was filtered in order to analyse the times for each task. It was assumed that the times for tasks done by the MFB (gluing, nailing, routing, and sawing) were relatively fixed, while the tasks that are done manually (layout of the joists, placing the sheathing, and tasks related to loading) would have more variation. Because of this, the total time for each of the MFB functions was collected in the time study and a distribution was created for each to represent the likely amount of time that the function would take. The manual tasks were then broken down into further individual tasks. The layout was broken down into the time to lay down each joist, nail the joists together, square the panel to the zero point in order for the MFB to be used, and other smaller tasks. Details of each task were also recorded, such as how many people were handling a joist, and whether or not it needed to be rotated or not. The layout 
of the sheathing was broken down to the time spent attaching the sheathing, as well as the time spent walking to and from the panel to bring the sheathing board to the panel and to return to get the next board. The loading prep was broken down into the time to attach each of the four straps to the panel, hook up each of the straps to the crane, and adjust the chains, if necessary.

The simulation model was built to match the order of operations carried out by the construction workers in the floor area. The results for the average simulation time generated from the model compared to the average time calculated from the time study can be seen in Table 1, along with the total time to build a panel and the average number of panels that are produced each day. The layout of the simulation model can be seen in Figure 2.

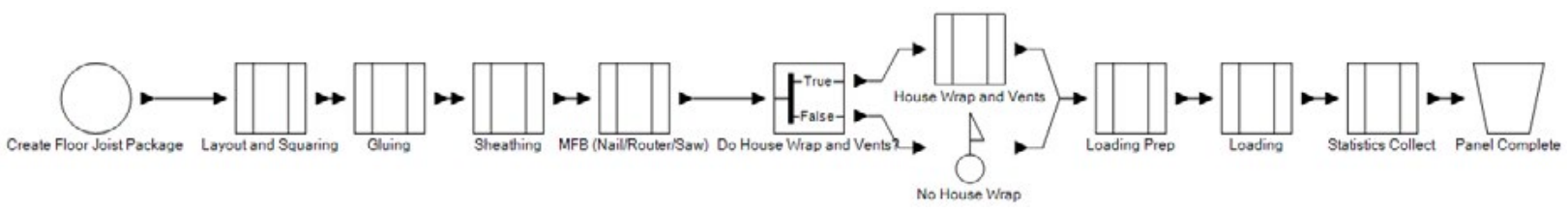

Figure 2. The layout of the current simulation model.

Table 1. The actual and simulated average task times for the tasks required in the construction of a floor panel. The actual times are based on data collected from approximately 50 panels.

\begin{tabular}{lccc}
\hline \multicolumn{1}{c}{ Task } & $\begin{array}{c}\text { Average Actual } \\
\text { Task Time (minutes) }\end{array}$ & $\begin{array}{c}\text { Average Simulated } \\
\text { Task Time (minutes) }\end{array}$ & $\begin{array}{c}\text { Percent } \\
\text { Error }\end{array}$ \\
\hline Joists Layout & 23.7 & 23.4 & $-1.3 \%$ \\
Gluing & 3.9 & 3.9 & $0.0 \%$ \\
Sheathing & 11.2 & 11.4 & $1.8 \%$ \\
MFB (Nail/ Router/Saw) & 18.3 & 18.3 & $0.0 \%$ \\
House Wrap and Vents & 7.0 & 7.4 & $5.7 \%$ \\
Loading Prep & 4.6 & 4.8 & $4.3 \%$ \\
TOTAL & $\mathbf{6 8 . 7}$ & $\mathbf{6 9 . 2}$ & $\mathbf{0 . 7 \%}$ \\
\hline
\end{tabular}

Validation and Verification. The simulation model can be validated by comparing the task time generated by the simulation model, which is generated by the timed sub-tasks combined to make up the total task time, and comparing this to the actual total task time that was collected during the time study. This comparison can be seen in Table 1. This comparison shows that the model is valid because the time to complete a panel is approximately equal to the actual time recorded to construct a panel. Also, the number of panels produced per day in the simulation model is approximately 13 , which is equal to the current production seen in the plant.

Future State Simulation Model. Based on the durations of the tasks and the observations made while recording them, several improvements are proposed in order to increase the rate of production at the floor panel area. These improvements include eliminating wasted movement, such as walking each sheathing board to the panel or rotating the individual joists more than 180 degrees, and eliminating wasted time, such as waiting for the machines or the crane. These improvements and their corresponding production increase per shift can be seen in Table 2 . It is important to note that all of these production increases are based on the one improvement being made to the base case, and applying several improvements will not necessarily result in the 
production increase being equal to the sum of the increases listed here, but will give a reasonable estimate.

Table 2. Expected production increase from the current state for proposed changes to the floor production based on simulation.

Proposed Change to Floor Production

\section{Eliminate joist rotation}

Glue manually when the MFB is in use

Always glue manually

Pre-install or eliminate house wrap and vents

Add another worker (4 total)

Sheathing transport solution

\section{Study of Indirectly Productive Tasks}

After the study of the productive tasks was carried out, it was noted that while improving the actual construction time by employing more efficient practices will increase the productivity of the floor construction area, an investigation into the delays and indirectly productive tasks will provide a greater opportunity to increase the proportion of the day that is spent on productive activities. Indirectly productive tasks are classified as tasks which do not directly contribute to the construction of the panel, but are usually tasks that still need to be done. These are not all necessarily non-value added activities, but are activities that can be done at different times or by different people, or possibly even reduced or eliminated. The fraction of the total man-hours per day spent on indirectly and directly productive activities can be seen in Figure 3.

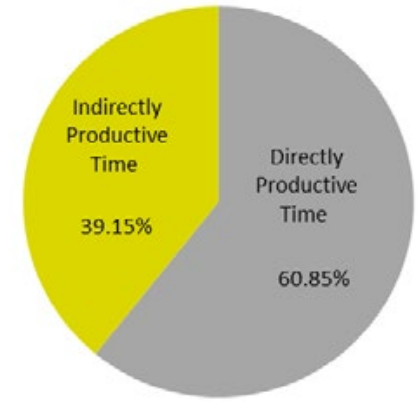

Figure 3. The average fraction of the total man hours spent per day on indirectly productive activities and directly productive activities at the floor panel construction station.

As was noted before, the time spent doing activities not classified as directly productive, which in this case include getting blocking from another location, sweeping, and monitoring the MFB, are also important when considering the overall effectiveness of an area in the factory. The focus of this portion of the study was on how the employees were spending their time. The background state of the panel construction was also recorded for these times in order to determine the following: (1) if there is no work being done to the panel (the table was idle); (2) if there is only one framer left when there could have been two; and (3) if the MFB is working, or the crane is working while these activities are being done. A breakdown of the average time spent per framer per day on each classification of indirectly productive activities can be seen in Figure 4. Figure 5 
shows the time spent carrying out indirectly productive activities during different background activities, or the level of production being done on the panel simultaneously.

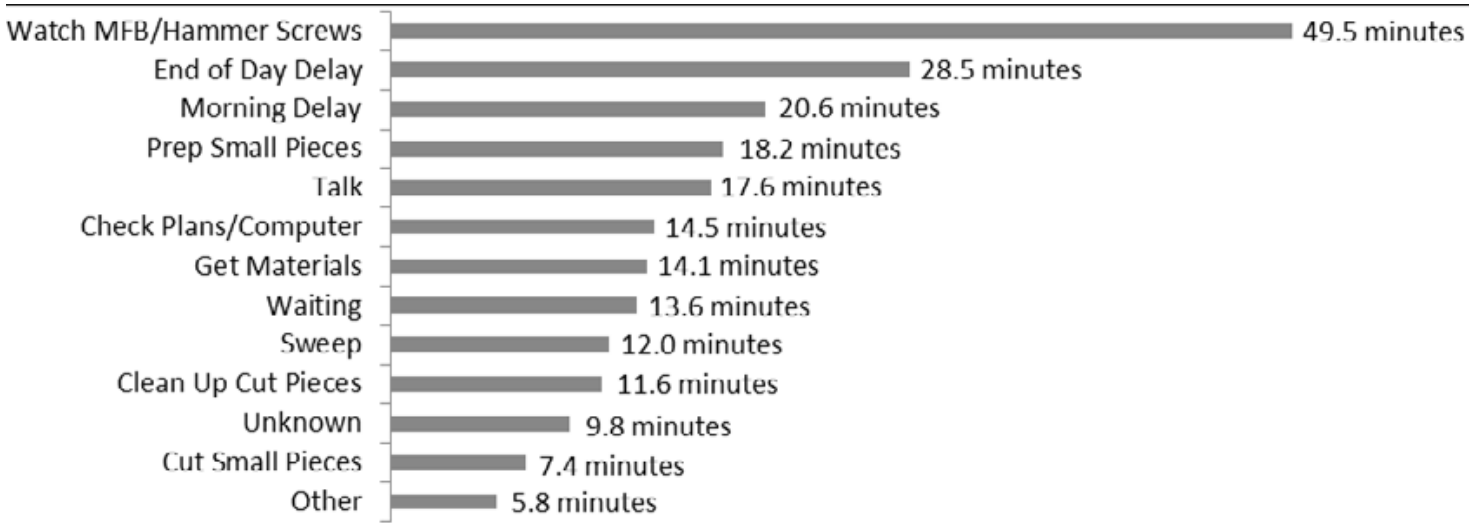

Figure 4. The average minutes per framer per day spent doing various indirectly productive activities.

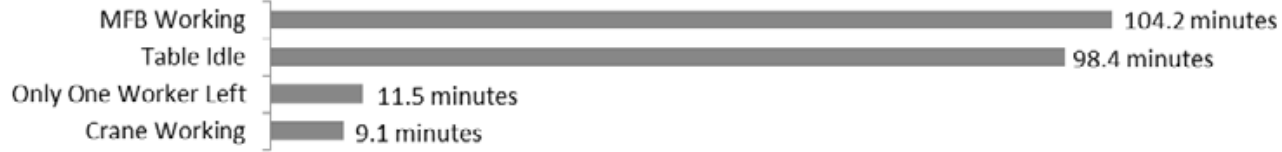

Figure 5. The average minutes per framer per day spent doing indirectly productive activities during different states of panel construction.

\section{RESULTS AND DISCUSSION}

\section{Results of the Study of Directly Productive Tasks}

From the original study of the directly productive activities, several changes to the work practices are identified as possible ways to increase the productivity of the floor production. These changes are identified in Table 2, along with the corresponding expected change in production. While some changes are more practical than others or would require more time and money to implement, the management can judge, with input from the employees, which changes they want to implement.

Eliminating the joist rotation refers to organizing the joists before they get to the floor panel in such a way that they never need to be rotated more than 90 degrees. The rotation is currently necessary to properly align the pre-drilled holes for vents in the floor joists. In order to eliminate the need to rotate the joists, the required direction of the floor joist will be determined while the joists are constructed and packaged, and they will be aligned in such a way to eliminate the need for rotation. Another wasted movement is the walking required to retrieve the sheathing and then to place it on the panel. Possible solutions to eliminate or reduce this time could include evaluating the location of the sheathing storage or using a material delivery bridge.

The multi-function bridge (MFB) works on each panel at two different times. The first time it applies glue to the top of the joists before the sheathing is placed, and the second time the 
sheathing boards are nailed on; then holes are routed in the sheathing in order to install the straps for the crane to lift the panel and the extra sheathing is trimmed off. While the machine is highly accurate and efficient for the second set of jobs, the time it takes to apply the glue is approximately equal to, or even greater than, the time it takes for the glue to be applied manually. By applying the glue manually at times when the MFB is in use at the other table, any time waiting for glue is eliminated. Also, if there are two framers working at the table, one can start bringing the sheathing to the table while the other completes the gluing, which is not possible when the MFB is used for gluing. This benefit can be realized even more if the gluing is always done manually, which will further reduce the waiting time for the MFB, since it will then only be required one time for each panel.

The installation of the house wrap and vents is another task that can be eliminated or done before the joist gets to the floor tables. Finally, adding another framer will increase production, and allow for some tasks to be done in a more ergonomically friendly manner, like using teams to lift heavy joists. As can be seen in Table 2, adding all of these recommendations to the floor construction process will result in an increase in production of almost three panels per day.

\section{Results of the Study of Indirectly Productive Tasks}

The study of the indirectly productive tasks shows that there is time where there is no work being done on a panel because the framers are working on these tasks instead. This time is where there is the most opportunity for improvement. Ideally, all of these tasks are completed during the time when the MFB is working, and never while the table is idle. In order to accomplish this additional planning, and possibly some assistance from workers nearby to do tasks such as prepare the blocking and lateral bracing, will be needed. There also needs to be a shift in thinking to reduce the morning and end of day delays. While some activities during this time are important and unavoidable, like morning safety meetings, most are material preparation and cleaning that should be done during the course of the day instead. Thinking about the work days as a continuous opportunity for work will help to reduce these delays, instead of thinking about them as individual days, where the framers will often not start a task if they do not have time to fully complete it at the end of the day. There is also a significant amount of time spent watching the MFB and hammering screws. While this is partially necessary to ensure proper operation of the MFB, it is not necessary to this extent and should be targeted to reduce by half.

If the goal of eliminating the time spent doing indirectly productive tasks while the table is idle was reached, about 4.7 additional man-hours would be available each day. As was shown in Table 1, it takes approximately 1.2 hours to complete a panel, with an average of 1.5 framers working on the construction, for a total of about 1.8 man-hours required to complete one panel. Eliminating the table idle time will therefore allow for an increase in production of about three panels per day

\section{Management and Worker Feedback}

The results of the time study and simulation were presented to members of the case study company, including upper management, production managers, and the workers on the line who build the floor panels. The feedback received was positive, and the group was able to point out where other issues may be created through any of the recommended process improvements, and also discuss any questions about any proposed changes. For example, it was pointed out that the 
reason that the joists may need to be rotated is because of the way machine operates. A solution of rotating the joists after the machine has cut the holes but before the joist package has reached the floor construction tables was attained. The group from the company decided to implement manual gluing when the MFB is busy, eliminating joist rotation at the tables, and are working toward eliminating the need for the house wrap and vents to be installed on the tables by adding an additional station dedicated to these tasks in order to decrease the takt time of the floor construction area. The feedback from the company on the changes implemented so far has been positive, and they have noticed a production increase in the floor production area.

\section{CONCLUSION}

By implementing the recommendations in this report, it can be estimated that the floor construction area can increase their production from the current 13 panels per day to almost 19 panels per day. This production increase would allow the factory to almost completely eliminate the night shift that is required in order to keep up when other areas of the plant are in full operation, but may require a fifth shift added each week at true maximum production, while further observation and analysis is done in order to identify further opportunities for productivity improvements. Production should also be further studied after the first recommendations are implemented to confirm assumptions about any proposed changes and help to mitigate any unforeseen outcomes that the changes could bring, as well as to evaluate future improvements.

\section{ACKNOWLEDGEMENTS}

The authors would like to thank everyone at the ACQBuilt factory in Edmonton, AB, Canada, for allowing us to observe the actions at the factory, both in person and through cameras, in order to collect the data required for this study. We would specifically like to thank Curt Beyer, Michael Loowell, and Christina Law for inviting us to do this study and organizing our visits, Darryl Stein for setting up and moving the cameras used to collect video of the process, and Sadiq Atlaf for providing feedback and direction to further the study. The research is funded by the NSERC Industry Research Chair Grant.

\section{REFERENCES}

AbouRizk, S., and Mohamed, Y. (2000). "Simphony-an integrated environment for construction simulation.” 2000 Winter Simulation Conference Proceedings (Cat. No.00CH37165), 2, 1907-1914.

Ikuma, L., Nahmens, I., and James, J. (2011). "Use of safety and lean integrated kaizen to improve performance in modular homebuilding." Journal of Construction Engineering and Management. American Society of Civil Engineers

Inyang, N., Han, S., Al-Hussein, M., and El-Rich, M. (2012). "A VR model of ergonomics and productivity assessment in panelized construction production line." Construction Research Congress.

Moghadam, M. (2014). "Lean-mod: An approach to modular construction manufacturing production efficiency improvement." University of Alberta. 